\title{
Descripción del dolor en pacientes con cáncer avanzado atendidos en los hospitales Carlos Van Buren y Eduardo Pereira, 2003-2004
}

\author{
ANDREA MARTINEZ $^{(1)}$ y JORGE GREGOIRE ${ }^{(2)}$
}

\section{RESUMEN}

Es un estudio de cohortes histórico de 400 pacientes del Programa de Alivio del Dolor, controlados en los policlínicos de los hospitales Carlos van Buren y Eduardo Pereira de Valparaíso entre enero 2003 y diciembre del 2004. Se buscó la información en los tarjetones de control, hojas de ingreso y egreso de los pacientes del programa. Con la información disponible, se confeccionó una base de datos con todas las variables a estudiar, las cuales se analizaron con el programa estadístico G-Stat.

Busca conocer las características epidemiológicas y la variación de la intensidad del dolor de los pacientes del programa de alivio del dolor por cáncer avanzado del Servicio de salud Valparaíso - San Antonio.

La mayoría de los pacientes tiene entre 45 a 64 años; el cáncer mas frecuente fue el gástrico (14.75\%); los EVA más bajos se encontraron en etapa I $(71,42 \%)$ y en etapa II $(66.6 \%)$; el $33 \%$ permaneció entre 0-30 días y el 6.75\% superó el año. El 62\% de la población estudiada en tratamiento analgésico percibió una disminución del dolor, el 14.25\% advirtió aumento en su EVA y el $17.75 \%$ no experimento cambio en su nivel del dolor.

El perfil epidemiológico es similar al de países en desarrollo, y los valores de EVA muestran un porcentaje de pacientes donde el dolor no se controló.

Palabras clave: dolor, cuidados paliativos, cáncer terminal.

\begin{abstract}
DESCRIPTION OF PAIN AMONG PATIENTS WITH ADVANCED CANCER ATTENDING THE CARLOS VAN BUREN AND EDUARDO PEREIRA HOSPITALS BETWEEN JANUARY 2003 AND DECEMBER 2004.

The present is a retrospective cohort study of 400 patients from the Pain Relief Program, followed-up at the outpatient clinics of the Carlos van Buren and Eduardo Pereira Hospitals located in Valparaíso between January 2003 and December 2004. Data were collected from the follow-up charts and the admission and discharge report sheets from patients within the program. A database was developed with the available data, and it included all the outcomes which were analyzed through the statistic program G-Stat.
\end{abstract}

(1) Departamento de Kinesiología. Facultad de Medicina. Universidad de Valparaíso. Tegula 370. Depto. 307. Viña del Mar. Chile. andrea.martinez@uv.cl

(2) Universidad de Valparaíso, Facultad de Medicina. Departamento de Salud Pública. Valparaíso. Chile. 
Purpose: To know the epidemiological features and the variations in pain intensity among the patients from the program of advanced-cancer-related-pain relief at the Valparaiso-San Antonio Health Service.

Most of the patients is aged between 45 and 64; the most common cancer was gastric cancer (14.75\%); the highest VAP were found at stage I $(71,42 \%)$ and stage II $(66.6 \%) ; 33 \%$ remained for $0-30$ days and $6.75 \%$ more than one year. Sixty-two percent (62\%) of the study population that were on analgesic therapy, perceived a decrease of pain, $14.25 \%$ noticed an increase in their $V A P$ and $17.75 \%$ did not experience any change at their pain intensity.

The epidemiological profile is similar to that of developing countries, and VAP figures show a percentage of patients in whom the pain was not followed-up.

Keywords: pain, palliative care, terminal cancer

\section{INTRODUCCIÓN}

El paulatino envejecimiento que ha experimentado la población chilena ha modificado el perfil epidemiológico, adquiriendo una creciente importancia las enfermedades crónicas no trasmisibles caracterizadas por periodos de incubación prolongados y por la presencia de factores de riesgo tales como; sedentarismo, sobrepeso, obesidad, dislipidemia, hábito tabaquito, consumo inmoderado de alcohol y drogas ${ }^{4}$.

A partir de 1994, se conforma la Comisión Nacional para el Alivio del Dolor por Cáncer y Cuidados Paliativos, con la participación del área pública y privada con la colaboración de la Asociación Chilena para el Estudio del Dolor. En el año 2003, el Programa de Alivio del Dolor y Cuidados Paliativos, pasa a ser parte de los programas con garantías explicitas de salud GES, a nivel nacional ${ }^{3}$.

La relevancia de estos programas se debe al creciente aumento de la esperanza de vida de los chilenos consecuente con el aumento de las tasas de morbi-mortalidad por tumores malignos. En Chile las tasas de mortalidad por esta causa aumentaron su frecuencia de 108.1 por cien mil habitantes en 1990 a 123.7 en el 20042. El 55\% de las personas con diagnostico cáncer actualmente requerirá estas atenciones ${ }^{8}$.

Dada la creciente importancia del Programa de Alivio del Dolor y Cuidados Paliativos, que procuran mejorar la calidad de vida de los enfermos y sus familias en torno a equipos de salud multidisciplinarios y comprometidos, se precisa conocer en detalle las características epidemiológicas y la intensidad del dolor de los pacientes con cáncer terminal que han participado del Programa de Alivio del Dolor en el Servicio de Salud Valparaíso - San Antonio, controlados en los policlínicos del dolor de los hospitales base; Carlos Van Buren y Eduardo Pereira.

\section{MATERIAL Y MÉTODO}

Se utilizó un estudio de cohortes histórico, de los pacientes del Programa atendidos de enero del 2003 a diciembre del 2004 en el Servicio de Salud Valparaíso - San Antonio. La muestra estuvo constituida por 400 pacientes que se controlaron en los policlínicos del dolor en los hospitales Carlos Van Buren y Eduardo Pereira, los cuales habían fallecido al momento de realizarse el estudio.

\section{Variables consignadas}

-Sexo

-Edad: número de años de vida registrado en ficha, en años cumplidos al momento del ingreso.

-Localización del cáncer: todo cáncer diagnosticado por médico, registrado en ficha clínica y clasificada según C.I.E - 10.

-EVA: Escala visual análoga de evaluación de la intensidad del dolor.

-EVA Inicial: Escala visual análoga de evaluación de la intensidad del dolor que se realiza al ingreso en el programa, que va del 0 al 10.

-EVA Final: Escala visual análoga de evaluación de la intensidad del dolor que se realiza al egreso en el programa que va del 0 al 10. 
- Diferencia de EVA: EVA inicial menos EVA final.

- Fármacos: Medicamentos utilizados por los pacientes del programa, según etapa de la escala analgésica de la OMS.

- Escala analgésica de la OMS: Tiempo de permanencia en el programa, días desde que el paciente ingresa al programa hasta que egresa.

\section{Recolección de datos}

La información fue recogida de los tarjetones de control y hojas de registro de egreso e ingreso al programa.

\section{Análisis estadístico}

Tablas univariadas, se observó las frecuencias absolutas y relativas de los tipos de cáncer, edad, sexo, EVA inicial y EVA final.

Se elaboraron tablas bivariadas de los valores de EVA al ingreso, al egreso y las diferencias de EVA de los pacientes del PAD según etapas de tratamiento analgésico para observar las variaciones del dolor.

Se realizó un análisis bivariado anova utilizado para crear una tabla de doble entrada para ver promedios de días tratamiento según diferencias de EVA.

\section{RESULTADOS}

En la Tabla 1, se observa que la mayoría de los pacientes de la muestra tenía sobre 65 años y era de sexo masculino, habiendo un número importante también entre los 45 y 64 años.

Tabla 1. Distribución por sexo y edad de los pacientes del PAD

\begin{tabular}{|l|cc|c|}
\hline EDAD & Sexo & TOTAL \\
\hline 20-44 años & 9 & 12 & 21 \\
\hline 45-64 años & 71 & 68 & 139 \\
\hline 65 y + años & 102 & 134 & 236 \\
\hline Total & 182 & 214 & 396 \\
\hline
\end{tabular}

Hubo 4 pacientes de los cuales no hubo información de sexo y edad.
En la Tabla 2, se observa que el cáncer más prevalente fue el gástrico (14.75\%), seguido del pulmonar $(14.25 \%)$ y el de próstata $(8.75 \%)$.

Tabla 2. Tipos de Cáncer del Programa de Alivio del Dolor (PAD) en los policlínicos del dolor de HCVB y Hospital Eduardo Pereira en los años 2003 y 2004

\begin{tabular}{|l|c|c|}
\hline $\begin{array}{l}\text { TIPOS } \\
\text { DE CANCER }\end{array}$ & N & \% \\
\hline Gástrico & 59 & 14.75 \\
\hline Pulmonar & 57 & 14.25 \\
\hline Próstata & 35 & 8.75 \\
\hline Vesicular & 26 & 6.5 \\
\hline Colon & 23 & 5.75 \\
\hline Mama & 22 & 5.5 \\
\hline Otros & 178 & 44.55 \\
\hline Total & 400 & 100 \\
\hline
\end{tabular}

En la Tabla 3, respecto al EVA inicial, se muestra que el $42.5 \%$ tuvo a su ingreso EVA entre 5 y 8 , y sólo un 17.5 superó el valor 9 como percepción de su dolor.

Tabla 3. EVA inicial de los pacientes del PAD atendidos en los policlínicos del dolor de los hospitales Carlos Van Buren y Eduardo Pereira en los años 2003 y 2004

\begin{tabular}{|l|c|c|}
\hline $\begin{array}{l}\text { EVA } \\
\text { INICIAL }\end{array}$ & $\mathbf{~ N}$ & $\mathbf{\%}$ \\
\hline $0-4$ & 122 & 30.5 \\
\hline $5-8$ & 170 & 42.5 \\
\hline $9-10$ & 70 & 17.5 \\
\hline Sin información & 38 & 9.5 \\
\hline Total & 400 & 100 \\
\hline
\end{tabular}

En la Tabla 4, del EVA final, se observa que el $62 \%$ egresó con dolor leve y un $4.25 \%$ con dolor severo.

En la Tabla 5, al examinar las diferencias de EVA, se encuentra que hubo un $14.25 \%$ de personas que aumentó su percepción del dolor, un $17.75 \%$ no percibió cambio y un $23.25 \%$ tuvo una variación sobre 5 puntos en su EVA al egreso. 
Tabla 4. EVA final de los pacientes del PAD atendidos en los policlínicos del dolor de los hospitales Carlos Van Buren y Eduardo Pereira en los años 2003 y 2004

\begin{tabular}{|l|c|c|}
\hline $\begin{array}{l}\text { EVA } \\
\text { FINAL }\end{array}$ & N & \% \\
\hline $0-4$ & 248 & 62 \\
\hline $5-8$ & 106 & 26.5 \\
\hline $9-10$ & 17 & 4.25 \\
\hline No se sabe & 29 & 7.25 \\
\hline Total & 400 & 100 \\
\hline
\end{tabular}

Tabla 5. Diferencias de EVA (EVA inicial menos EVA final) de los pacientes del PAD atendidos en los policlínicos del dolor de los hospitales Carlos Van Buren y Eduardo Pereira en los años 2003 y 2004

\begin{tabular}{|l|c|c|}
\hline $\begin{array}{l}\text { DIFERENCIAS } \\
\text { DE EVA (DEVA) }\end{array}$ & $\mathbf{N}$ & \% \\
\hline-8 a -1 & 57 & 14.25 \\
\hline 0 & 71 & 17.75 \\
\hline 1 a 4 & 119 & 29.75 \\
\hline 5 a 8 & 73 & 18.25 \\
\hline 9 a 10 & 20 & 5 \\
\hline No se sabe & 60 & 15 \\
\hline Total & 400 & 100 \\
\hline
\end{tabular}

En la Tabla 6, se aprecia que los EVA más bajos al egreso se encontraron en etapa I con un $71.42 \%$ y etapa II con un $66.6 \%$. Las mayores variaciones de EVA se encontraron en etapa II y III. La variación del EVA fue mayor en aquellos que usaron morfina de 3.68 , respecto a los que no la usaron 2.22. Pero estos valores con un $\mathrm{p}>0.05$ no fueron estadísticamente significativo.

En la tabla 7, el 33\% de los pacientes permanece entre 0 y 30 días en el PAD, y un $18.50 \%$ sobre 180 días, un pequeño porcentaje $(6.75 \%)$ superó el año.

Tabla 7. Días de permanencia de los pacientes del PAD

\begin{tabular}{|l|c|c|}
\hline DÍAS & $\mathbf{N}$ & \% \\
\hline $0-30$ & 132 & 33 \\
\hline $31-90$ & 111 & 27.75 \\
\hline $91-180$ & 79 & 19.75 \\
\hline $181-365$ & 47 & 11.75 \\
\hline $365 \mathrm{y}+$ & 27 & 6.75 \\
\hline Se desconoce dato & 4 & 1 \\
\hline Total & 400 & 100 \\
\hline
\end{tabular}

En la Tabla 8, se encontró una relación significativa entre las variaciones del EVA y los días de permanencia en el PAD. También se muestra que en los pacientes que aumentó el dolor, el promedio de permanencia fue de 14.51 días y en los que el E.V.A. disminuyó de ocho a diez puntos el promedio fue de 144.04 días.

Tabla 6. Etapas de tratamiento analgésico (OMS) según diferencias de EVA

(EVA al ingreso menos EVA al egreso del PAD)

\begin{tabular}{|l|c|c|c|c|c|c|c|c|c|c|}
\hline DEVA & $\begin{array}{c}\text { Etapa 0 } \\
\mathbf{n}\end{array}$ & $\mathbf{\%}$ & $\begin{array}{c}\text { Etapa I } \\
\mathbf{n}\end{array}$ & $\mathbf{\%}$ & $\mathbf{n}$ & $\mathbf{6}$ & $\begin{array}{c}\text { Etapa III } \\
\mathbf{n}\end{array}$ & $\mathbf{\%}$ & $\begin{array}{c}\text { Total } \\
\mathbf{n}\end{array}$ & $\mathbf{\%}$ \\
\hline$-8 \mathrm{a}-1$ & 4 & 13.3 & 1 & 7.14 & 25 & 13.44 & 27 & 15.88 & 57 & 14.25 \\
\hline 0 & 6 & 20 & 4 & 28.57 & 34 & 18.27 & 27 & 15.88 & 71 & 17.75 \\
\hline $1-4$ & 5 & 16.6 & 6 & 42.85 & 59 & 31.72 & 49 & 28.82 & 119 & 29.75 \\
\hline $5-8$ & 2 & 6.6 & 1 & 7.14 & 29 & 15.59 & 41 & 24.11 & 73 & 18.25 \\
\hline $9-10$ & 1 & 3.3 & & 0 & 14 & 7.52 & 5 & 2.94 & 20 & 5 \\
\hline No se sabe & 12 & 40 & 2 & 14.28 & 25 & 13.44 & 21 & 12.35 & 60 & 15 \\
\hline Total & 30 & 100 & 14 & 100 & 186 & 100 & 170 & 100 & 400 & 100 \\
\hline
\end{tabular}


Tabla 8. Promedio de los días de tratamiento según diferencias de EVA

\begin{tabular}{|l|c|c|}
\hline $\begin{array}{l}\text { DIFERENCIAS } \\
\text { DE EVA }\end{array}$ & $\begin{array}{c}\text { DÍAS DE } \\
\text { PERMANENCIA } \\
\text { N }\end{array}$ & Promedio \\
\hline-8 a -1 & 59 & 14.51 \\
\hline 0 & 71 & 55.05 \\
\hline 1 a 2 & 64 & 96.04 \\
\hline 3 a 4 & 53 & 130.3 \\
\hline 4 a 5 & 40 & 152.73 \\
\hline 6 a 7 & 42 & 148.54 \\
\hline 8 a 10 & 40 & 144.04 \\
\hline Total & 339 & 132.69 \\
\hline
\end{tabular}

Se desconocieron 61 datos.

\section{DISCUSIÓN}

El cáncer más frecuente en los paciente en esta investigación, fue el gástrico $(14,75 \%)$, seguido del pulmonar $(14,25 \%)$ y el prostático $(8.75 \%)$.

El rango de edad de la muestra fue de 22 a 98 años y la mayoría era mayor de 65 años, siendo el $52.02 \%$ hombres, lo cual coincide con otros estudios en CP que muestran que la edad promedio de los usuarios de estas atenciones supera los 60 años, artículos australianos informan de una edad promedio de 65.5 años y artículos europeos un promedio de 66 años $^{3,5}$.

Respecto al EVA, el $60 \%$ de los pacientes ingresó con dolor de moderado a severo y el $62 \%$ egresó con dolor leve. Al analizar en detalle las diferencias en el dolor, se observó un grupo de pacientes durante su periodo de permanencia en el programa el dolor aumento $(14,25 \%)$ y en un $17,75 \%$ no tuvo variaciones en la percepción del dolor durante su tiempo en el programa.

En relación a este tema, Cleeland y col. estudiaron la intensidad del dolor en 1308 pacientes ambulatorios con cáncer metastático, y observaron que el $42 \%$ no recibió una terapia analgésica adecuada. Otros trabajos europeos han observado que hay una alta frecuencia de pacientes que soportaron dolor de moderado a severo en etapa III de tratamiento analgésico, lo que sugiere que no recibieron dosis adecuadas de tratamiento. Este mismo estudio concluyó que de los pacientes que usaron morfina, sólo unos pocos necesitaron dosis altas 6 .

Al estudiar los cambios de EVA se observó que los mayores descensos se produjeron en etapa II y III. Pero también en etapa III fue donde se observo el mayor aumento en la percepción del dolor en los pacientes del PAD.

El tiempo de permanencia en $\mathrm{CP}$, varió de un día a 1131 días, de los cuales el $60.75 \%$ de la población estudiada permaneció un periodo inferior a tres meses, aunque hay un $18.5 \%$ que superó los 180 días lo cual difiere de otros estudios nacionales en que sólo el $8.4 \%$ supera esa cantidad de días pero realizados con muestras inferiores a cien pacientes ${ }^{2}$. Investigaciones en poblaciones italianas mostraron una media de supervivencia desde el ingreso a cuidados paliativos de 234 días (103 - 374 días $)^{1}$.

En relación al EVA final, la variabilidad del dolor y los tiempos de permanencia, se observó asociación estadísticamente significativa $(\mathrm{p}<$ $0.05)$ y se puede ver que los días de permanencia aumentan en la medida que el dolor se controla.

\section{AGRADECIMIENTOS}

A Danny Casanova, Estadístico Universidad de Valparaíso, Departamento Salud Pública; a Pablo Cáceres, académico Universidad Católica de Valparaíso, y a las personas del Programa de Alivio del Dolor, en especial a las de los centro que me permitieron trabajar con sus datos y las enfermeras por llevar todo registrado.

\section{REFERENCIAS}

1. CONSTANTINE. M, BECARRO. M, MERLO. F; The last three months of life of Italian cancer patients. Methods sample characteristics and response rate of the Italian survay of the dying of cancer; Palliative Medicine 2005; 19: 628 -638.

2. DEIS MINSAL, Indicadores básicos de salud, Octubre 2006.

3. EBNER P, CUEVAS C, SWETT E, YENTZEN; Programa de alivio del dolor por cáncer avanzado y cuidados paliativos Llay-Llay de enero 2001- febrero 2004; G.Rev. Chilena. Salud Pública 2005; Vol.9 (1):25-31

4. GLARE. A.P, EYCHMUELLER. S, MCMAHON. P; Diagnostic accurancy of palliative pronogtic score in hospitalized patients with advance cancer"; Journal 
of Clinical Oncology; Diciembre 2004: Vol. 22; No 23: 4823-4828

5. JUDUE L, VEGA M, ESCOBAR M, DELGADO I, GARRIDO C, LASTRA P, ESPEJO F, PERUGA A; "Factores de riesgo para enfermedades no trasmisibles: metodología y resultados globales de la encuesta de base del programa CARMEN (Conjunto de acciones para la reducción multifactorial de las enfermedades no trasmisibles)". Minsal, OPS Junio 1999.

6. KLEPSTAD. P., KAASA. S., CHERNY. N., HANKS. G., DE CONNO. F; Pain and pain treatments in european palliative care units across sectional survey from European association for palliative care research network; Palliative Medicine 2005: 19: 477 - 484.

7. MINSAL. Norma Técnica del programa de alivio del dolor y cuidados paliativos, 2004.
8. MINSAL. Departamento de enfermedades crónicas, Unidad de cáncer; Manual: ¿Cómo cuidar a las personas con cáncer avanzado en el nivel primario de atención?; 1 Ed., 2005.

9. ROSEWAX. L.K, MCNAMARA. B., BLACKMORE. A.M., HOLMAN. C.; Estimating the size of a potencial palliative care population; Palliative Medicine 2005; 19: 556 - 562.
Recepción: 19 octubre 2007 Aprobación: 17 de marzo 2008

Usted puede comentar éste y otros artículos publicados en la Revista Chilena de Salud Pública, enviando un correo electrónico a revistasp@med.uchile.cl 